\title{
Epigenetics: A new way to understand and tackle cancer
}

\author{
M. Bonucci*
}

\author{
Chief of Clinical Pathology Department, Oncology Outpatient, San Feliciano Hospital, Italy
}

In view of understanding the causes of cancer you need to consider all possible causes. Until now, the most accredited theory responds to an alteration of the DNA that leads to the uncontrolled proliferation of a cell. What is not well understood, however, is the reason why this alteration occurs and why it continues to be present. We all think that the mutation is undetermined and that for "unclear reasons" the mutation becomes stable and persistent with the expression of the mutation, RNA and protein synthesis, of the alteration and subsequent progression towards the neoplasm. So far it is the knowledge closely linked to DNA damage and until now we are moving to know more and more DNA damage that leads to the neoplasia [1]. We know that some of these damages are caused by viruses, or by ionizing radiation or by carcinogens like benzene or many other chemicals. Until today, medical science has discussed precisely the importance of DNA in the determinism of diseases and consequently the possibility of predicting and preventing them by codifying the structure. But this did not bring the expected results. Neoplastic diseases are on the increase, without finding great results in the medical field (if not a better quality of life and a longer disease-free survival). Starting from a fundamental concept that concerns the knowledge of our DNA, we can consider this double strand the result of millions of years of continuous remodeling given above all by the plasticity of DNA to vary depending not only on chance (meeting between male and female DNA) but to the continuous adaptive response to the surrounding environment. The evolution of the various species (bacteria, multicellular organisms, animals) present on the earth are linked precisely to the change of the environment and to the adaptation of the single organism to the environment itself. We cannot live in an oxygen-free environment while some bacteria can do it. It means that the environment can modulate the expression of its DNA depending on where it lives and what it comes into contact with. A new paradigm of life is this: an environment that modulates the expression of DNA. If we think that a bee larva becomes a queen bee only if it is fed with royal jelly, it means that the decision of who to become a queen bee is of the group but the ability to make it become such is related to what they feed it, it is not a "casual" but "sought" birth [2]. So it's thinkable also in our case? Are we individuals who change depending on where and what we eat and breathe? All this thought has a name: EPIGENETICS. According to this science, the genes are entrusted with the task of maintaining the archive of our history, but it is thanks to the environment that surrounds them that they will be activated or not in their function of expression, of modification with the possible harmful evolution. DNA is nothing more than a large database,

Copyright: (C2018 Bonucci M. This is an open-access article distributed under the terms of the Creative Commons Attribution License, which permits unrestricted use, distribution, and reproduction in any medium, provided the original author and source are credited. a library, a hardwere, which contains billions of information, the result of billions of years of evolution [3]. Which are available and will prove useful when readers will use them. The database does nothing but enrich itself with new data, but it is only thanks to the ability of the reader that he will be able to put them in place. The destruction or tampering of a part of the database is unpredictable and rare (chromosomal or genetic damage) compared to the frequent use of a reader. Here we can begin to understand how a different gene expression is possible. The DNA can be modified either directly with methylation (addition or subtraction of methyl, small amino acid sequences) and through the modification of particular proteins, the histones, which envelop the DNA helix and determine its opening and expression, or through the production of small minor RNAs that are structures connecting the nucleus and the cell cytoplasm. Here then extracellular substances can intervene on these mechanisms and modify not the DNA but its expression (files are opened, books are not clear, data not known). And if we think of multiple substances used in all fields (pesticides, heavy metals, endocrine disruptors, benzene) we understand how they can interfere with DNA and modify or damage its expression. A function of the methyl groups is precisely to keep the oncogenes silent, their reduction or hyper-free expression mediated by free radicals could trigger a neoplastic pathology. This new way of looking at the environment as an important tool in the expression of our DNA is increasingly taking hold. This means that everything around us has a key role in our diseases and not least the neoplastic pathology. In the word environment it is meant all that surrounds us: the air we breathe, the food we eat, the thoughts we make. It is a new reading of the neoplasm, it is the reading that many should do, because the neoplasm is not a crazed, random cell, but a cell that has changed according to what it came into contact with. Changing the environment is the first and fundamental step in the case of neoplastic pathology [4].

\section{References}

1. Gerhauser C (2013) Cancer chemoprevention and nutriepigenetics: state of the art and future challenges. Top Curr Chem 329: 73-132. [Crossref]

2. Frazer IH (2018) Eradicating HPV-Associated Cancer Through Immunization: A Glass Half Full. Viral Immunol [Crossref]

3. Bishop KS, Ferguson LR (2015) The interaction between epigenetics, nutrition and the development of cancer. Nutrients 7: 922-947. [Crossref]

4. Wils LJ, Bijlsma MF (2018) Epigenetic regulation of the Hedgehog and Wnt pathways in cancer. Crit Rev Oncol Hematol 121: 23-44. [Crossref]

Correspondence to: $\mathrm{M}$ Bonucci, Chief of Clinical Pathology Department, Oncology Outpatient, San Feliciano Hospital, Italy, E-mail: maxbonucci@artoi.it

Received: January 03, 2018; Accepted: January 18, 2018; Published: January 22, 2018 